\title{
Die Gedichte des Guillem Augier Novella.
}

\section{Finleitung.}

Bartsch führt in seinem Grundrifs der provenzalischen Litteratur drei Dichter des Namens Augier an: Augier Novella (No.37), Auzer Figueira (42) und Guilhem Augier (205). Die Nichtexistenz des zweitgenannten ist durch Levys Dissertation: Guilhem Figueira (Berlin 1880) nachgewiesen. Die Identität der beiden anderen soll im Folgenden dargethan und das Leben und Dichten dieses einzig nachweisbaren Trobadors Augier ausführlich behandelt werden.

Ueber die Lieder, die Bartsch unter No. 37 und No. 205 stellt, und ihre Dichter ist von vielen, meist aber nur flüchtig in kurzen Citaten und Anmerkungen', gehandelt worden. So liefsen sich nennen: Millot, Hist. litt. des Troub. I 34 I, III 409 - Histoire littéraire de la France XIII 420, XVIII 550 - Crescimbeni, Hist. della volgar poesia II 202 - Diez, Altrom. Sprachdenkm. S. 1 24; Leben und Werke der Troub. 1882, 2. Aufl., Register Cavedoni in: Memorie della reale Acad. di scienze di Modena, tom. II, I858, p. 28 I - Revue critique I 868, II p. 2 I - Gior nale storico IX 205 - Azaïs, Troub. de Béziers, 2. éd. 1869, pag. I 19--127 - Mahn, Gedichte No.577 Anm. - Suchier in: Lemkes Jahrb. XIV 292 - Bartsch, Ztschr. II S. I97 - Maus, P. Cardinals Strophenbau, Verzeichnis, Ausg. u. Abhandl. (Stengel), Marburg, No. 5 - Thomas, Franc. Barberino et la litt. au moyenâge, p. 90 Anm. - P. Meyer in: Romania X 263 - O. Schultz in: Ztschr. VII 204; Briefe Raimbauts de Vaqueiras p. 73, 114; ital. Uebersetzung der Ausgabe (1898) p. 93, 150 - Chabaneau in: Hist. gén. de Languedoc p. p. De Vic et Vaissette, I885, t. X p. 296. 334. 339. 350 - Knobloch, Streitged. im Prov. u. Altfrz., Diss. Breslau 1886 - Selbach, Streitgedichte in der altprov. Lyrik, Ausg. u. Abh. (Stengel), Marburg, No. 57, p. 18 - Litteraturblatt für germ. u. rom. Phil. 1887, p. 78 - Wit thöfft, Sirventes joglarescs, Ausg. u. Abh. (Stengel), Marburg, No. 88 - Springer, Altprov. Planhs, Berliner Beitr. zur germ. u. rom. Phil. 1895, No. VII, p. 68.

Ausführlicher hat sich O. Schultz-Gora mit den Liedern beschäftigt in Ztschr. VII 198 und 204, IX 120 und Deutsche Litt.Zeitung 1887 col. 201. Er hat an diesen Stellen viele wertvolle Resultate mitgeteilt, welche im Folgenden sämtlich benutzt sind.

Was ich nach Studium der provenzalischen Grundlagen und obiger Litteratur für das Richtige halte, gebe ich im voraus kurz so an: 
I. Alle jene Namen in den Handschriften wie: Ogier, Augier Novella, Guilhem Augier, Guilhem de Bezers etc. meinen eine und dieselbe Person: den Joglar Augier aus S. Donat.

2. Dieser Augier lebte etwa von I 185 bis 1235 .

3. Er wirkte erst in Béziers, nach 1209 ging er nach Italien, wo er den Beinamen Novella erhielt.

Zunächst gilt es festzustellen, welche Gedichte mit Recht dem Augier zugeschrieben werden. Die Canzone 37, I Era quan liverns nos laissa stellt Bartsch mit Unrecht hierher, wie Schultz (Ztschr. VII I 8 I unter Albertet Cailla) schlagend nachgewiesen hat. Die Canzone bildet nämlich mit einer andern $(G r .174,2)$ eine Tenzone über die Frage, ob alte oder junge Frauen vorzuziehen seien. Die unsere ist von einem gewissen Albert (im Gedicht genannt Gaubert oder Albertet) Bernard de Durfort, die ihr entgegengesetzte von Bertran de Preissac. Zum Ueberflufs ist nun auch noch eine wirkliche Tenzone über denselben Gegenstand von denselben Personen vorhanden.

Also gehört 37, I unserm Augier nicht.

Andererseits kann das Descort Quan vey [No. 5] (Gr. 242, 6I) von dem Planh Quascus plor [No. 3] $(205,2)$ nicht getrennt werden; denn in beiden Liedern wird die Geliebte im Geleit mit bel papagai angeredet; Hs. $\mathrm{R}^{2}$ schreibt das Descort auch ausdrücklich Guilhem Augier zu, während $R^{1}$ und C es G. de Borneilh zuteilen. Schultz, Ztschr. VII S. 120 Anm. spricht es dem Augier ab, ohne einen Grund anzugeben.

Die übrigen Lieder unter Nr. 37 und 205 bei Bartsch sind in der That von Augier.

Gegen eine Scheidung von Augier Novella und Guilhem Augier spricht, dals das Lied 205, 5 von $R$ und $C$ reg. dem Guilhem Augier, von $\mathrm{C}$ selbst und $\mathrm{D}$ aber dem Augier Novella zuerteilt wird. Die Schreiber der Hss. kannten also keinen Unterschied.

Ferner giebt es blofs eine Lebensbeschreibung, die von einem Ogier ohne Beinamen redet. Ich gebe sie hier nach Chabaneau (Hist. g. d. Lang. Tom. X): Ogiers si fo un joglars de Vianes, qu'estec longtemps en Lombardia e fez bons descortz e fez sirventes joglarescs, que lauzava'ls uns e blasmava los autres. Sie findet sich in IK vor dem Sirventes Toz temps [No. I] (Gr. 37, 3).

Sirventes joglarescs von der Art, wie sie Witthöfft dem Begriffe untergelegt hat, sind uns von Augier nicht erhalten.

Hätten wir es wirklich mit zwei getrennten Personen im Sinne Bartschs zu thun, so wäre es seltsam, dafs wir, während wir genau wissen, dafs die beiden Sänger ungefähr gleichzeitig lebten, von dem einen kein Lied nach 1210 , von dem andern keins vor 1215 nachweisen könnten. Denn dafs der Planh $(205,2)$ wirklich auf den im Jahre 1209 im Kerker gestorbenen Vizgrafen Raimund

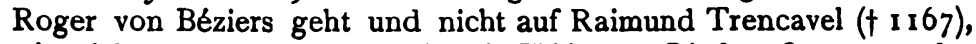
wie viele wollen, beweise ich mit Hilfe des Liedes Quan vey, das auch den bel papapai anredet. Die darin genannten Frauen Azalais 
de Boissazo und die Marquesa de Menerba glänzten um 1200. Andererseits hat Cavedoni gezeigt, und es ist auch leicht aus dem Lied zu ersehen, dafs das Sirventes Toz temps $(37,3)$ nicht vor 1 220, wo Kaiser Friedrich II. gekrönt wurde, entstanden ist. Friedrich I. Barbarossa ist durch den Namen Roger Frederic ausgeschlossen, der alte König Roger Frederic von Sizilien aber durch das Wort emperis ( Toz temps IV 32). Roger Frederic, der Kaiser, kann nur Friedrich II. sein, der, was wenig bekannt zu sein scheint, den Zunamen Roger hatte (vgl. Boehmer, Regesta Imper., 1881, V S. 154, Jahr I 196).

Alle oben genannten Schwierigkeiten klären sich auf, wenn wir einen einzigen Dichter annehmen, und kein Grund läfst sich gegen diese Identifizierung anführen. Mit Leichtigkeit lälst sich alles Gegebene zum Lebensbilde eines einzigen Mannes ordnen, einem Lebensbilde, das wir jetzt mit Hilfe der wenigen gegebenen Punkte uns zu entwerfen suchen wollen.

Guilhem Augier stammt aus Saint-Donat im Viennischen (jetzt ein kleiner Ort im Winkel zwischen Rhône und Isère unweit der Stadt Tournon, Dép. Drôme). Sein Geburtsjahr werden wir um oder auch vor I 185 setzen müssen; denn 1209 ist er schon einige Zeit in Béziers, hat aber auch schon einige Jahre hinter sich, in denen er sich als Buschritter mit Speer und Schild nach Art des Bertran versuchte (Tenzone No. 9). Dann hat er dieses Räuberhandwerk aufgegeben, hat Speer und Schild mit der Leier vertauscht und sucht sein Brot als Joglar an den Höfen der Grolsen des Landes.

Als das früheste Lied haben wir anzusehen das Descort Erransa (No. 4). Es ist in Béziers entstanden; denn es ist in derselben Hs. erhalten wie der Planh (No. 3) und, wie er, einmal dem Guilhem de Bezers, das andere Mal dem Guilhem Mogier de Bezers zugeschrieben. Es fällt vor I 209; denn in dem I 209 geplünderten und verwüsteten Béziers wird sich der Dichter nach dem Tode seines Gönners nicht so lange mehr aufgehalten haben, um dort noch Mufse für Liebesgedichte zu finden.

Augiers Liebesgeschichte vor I 209 erinnert sehr an die des Raimon de Miraval. Augier richtet das Descort No. 5 an Azalais de Boyssazo, den bel papagai, welcher Versteckname offenbar der gleichen Vokale (Azalais - papagais) wegen gewählt ist, erwähnt aber auch, da/s er der edeln Marquesa de Menerba Treue schuldig ist. Denken wir an Raimons Liebesgeschichte: Er besang zuerst die Marquesa de Menerba (offenbar dieselbe, die Augier meint), und zwar so herrlich, dals die Fürsten von Toulouse und Aragonien und auch der jugendliche Vizgraf von Béziers (erst 24 Jahre alt, I209) in Liebe zu der noch nicht gesehenen Frau entbrannten und sogleich Reiserüstungen trafen. Auch der Gegenstand des Descorts Erransa ist Liebe voll Eifersucht zu einem herrlichen Weibe, das der Dichter noch nicht gesehen, von dem er aber gehört hat. Dieses Motiv ist freilich ein Gemeinplatz in der provenzalischen Poesie, immerhin liefse sich denken, dafs es der Dichter bei jener 
Gelegenheit in eigener Begeisterung für die gepriesene Marquesa de Menerba oder im Auftrage seines verliebten Herrn verfafste.

Aus der Lebensgeschichte Raimons von Miraval ist bekannt, dafs Peire von Aragon die Liebe des schönen Weibes davontrug und dals der getäuschte Raimon seine Liebe und Lieder nun der jungen Azalais de Boyssazo auf Lombers zuwandte (Lombers liegt ebenso wie Menerba im Albigensischen, also nicht allzuweit von Béziers). Augier hat sich offenbar den Vorfall nicht sehr zu Herzen genommen. In No. 5 schwört er noch: per la fe qu'ieu dey a la pros Marquesa gaia de Menerba, a cuy sopley. Aber No. 5 selbst und auch No. 3 sind an eine andere, eben auch die Azalais de Boyssazo, gerichtet. No. 3 ist eine rührende Klage über des jugendlichen Gönners gewaltsamen Tod, für den unser Planh ein neues Zeugnis ist. (Vgl. P. Meyer, Chanson de la Crois. I 862, 3361 und II S. 46 Anm. 3.) Raimon Roger starb im November 1 209. Im Frühling 1210 scheint Augier schon von dem eroberten, geplünderten und seines geliebten Herrn beraubten Béziers Abschied genommen zu haben. Was konnte ihm auch noch blühen in dem vom Albigenserkriege überall aufs grausamste verheerten Lande? Das Frühlingsdescort No. 5 (Quan vey) ist wahrscheinlich sein Abschiedslied. Der Inhalt freilich zeigt nur, dafs er mit der verehrten Azalais nicht eben allzuweit gekommen ist. Nicht einmal sie anzureden hat er gewagt. Und doch hat sie sein Herz ihm ganz genommen. Am Schlusse aber spricht er unverhohlen die Namen der beiden Geliebten neben dem Senhal aus, den er 1209 im Planh allein anwandte, und fügt zweimal hinzu, dafs er Azalais nicht mehr sähe.

Auf jeden Fall war er schon vor 1212 in Italien. Denn er hat den Kaiser Friedrich II. noch als König gesehen (No. I, IV). Das muls vor 1212 gewesen sein. Denn 1212 bis 1220 weilte Friedrich II. in Deutschland und eilte von da aus 1220 stracks nach Rom, um sich zum Kaiser krönen zu lassen. Leicht möglich ist, dafs ihn Augier 1212 auf dem Durchzug durch Oberitalien nach Deutschland, wobei ihm der Markgraf von Monferrat das Geleite gab, kennen gelernt hat.

Mahn schliefst aus dem Sirventes No. I, II I 5 qu'anc piets no fo ros a Roma romieus, dals Augier in Rom gewesen sei. Doch bietet dafür dieser Vers nicht mehr Gewifsheit, als wenn man aus No. 7, $\mathrm{V} 42-43$ no volgr'aver Proenza ab tota Lombardia allein folgern wollte, dafs er in der Provence und Italien gelebt hätte. Gewils brauchen wir das Lombardia der Lebensnachricht nicht wörtlich zu nehmen, es kann ganz Italien gemeint sein. Doch beweist auch andererseits keine Stelle, dals er über Oberitalien hinaus nach Süden gekommen ist.

Ich nehme nun mit P. Meyer (Rom. X 202) an, dafs unser Joglar Augier identisch ist mit jenem Auzer, dessen Name in einer Tenzone zwischen Bertran d'Aurel, G. Figueira und Aimeric de Peguilhan und in einem Gedicht Sordels (s. Levy, G. Figueira, Berl. Diss. 1880) vorkommt, und dals die Tenzone No. 9 eben mit 
jenem Bertran d'Aurel gewechselt worden ist. Als Beweis dafür dient die Metrik von No. 9. Die Episode, die auf den Charakter unseres Joglar Augier ein Streiflicht wirt, hat sich danach etwa so abgespielt: ${ }^{1}$ Der Albigenserkrieg, der schon Augier des Gönners und der Heimat beraubt hatte, scheuchte besonders seit der Einnahme von Toulouse durch die Kreuzfahrer 1215 immer mehr Sänger über die Alpen. Die Tenzonen, die Levy in seiner Dissertation über Guilhem Figueira behandelt, deuten darauf hin, dafs sich in irgend einer der oberitalischen kleinen Residenzen eine ganze Anzahl solcher ausgewanderter Spielleute zusammengefunden hatten, so Figueira, Bertran d'Aurel, Aimeric de Peguilhan, Guilhem del dui Fraire und unser Augier, vielleicht auch Guilhem de la Tor und Sordel. Von Figueira wird uns berichtet, dals er nicht an den Höfen, sondern lieber in Kneipen verkehrte und Leute von den Höfen durch Spott reizte, wenn sie mit ihm zusammenkamen. Bertran d'Aurel, der ja mit Figueira tenzonierte, führte offenbar ein ähnliches Leben. Er stahl als Buschritter mit Gewalt und List Vieh, so dafs ihm die Bauern nachstellten. Augier wirft ihm das in der Tenzone No. 9 vor, aber wir hören gleichzeitig, dafs er selbst dies gefährliche, elende, aber freie Räuberleben vor seiner Joglarzeit auch versucht hatte. In der That steht seine Bildung auf nicht viel höherer Stufe als die jener Buschklepper, was uns übrigens auch der Satz in I 7 des Sirventes No. I: que no sui trop apres bestätigen kann. Denn während die übrigen mit dem Messer nach einander stielsen und sich Käse an den Kopf warfen (s. Levy, Guilh. Figueira), hat unser Augier dem Figueira einen Schwertstreich, der nicht ungefährlich gewesen zu sein scheint, mitten ins Gesicht gegeben. Bedenken wir, wie jene rohen Joglars in den Tenzonen sich Grobheiten sagten und dafs Figueira überhaupt gern Hofleute von Augiers Art durch Spott reizte, so finden wir eine solche Handlung wic jenen Schwertstreich begreiflich. O. Schultz setzt das Gedicht Sordels, worin auf den Schlag Augiers angespielt wird, etwa ins Jahr 1225 (Ztschr. 7, 204). Die obige Episode hätte sich dann nicht lange vor 1225 begeben. Ungefähr in dieselbe Zeit fällt wohl auch das Descort No. 6 Ses alegrage. Die in Vers $\mathrm{IO}_{4}$ genannte Dame Mil' en Romaigna identifiziert O. Schultz mit der Emilia von Ravenna, welche von Aimeric de Pegulhan, Guilhem de la Tor und Albert de Sestaron besungen wird (Ztschr. 7, 201 Anm. und 229; 9, I 7 Anm. 3).

In der erwähnten Tenzone No. 9 preist Augier seine Stellung als Joglar in den Häusern der Grofsen mit den Worten: "Joglars sind keine Knechte, sondern sie suchen Barone und fröhliche Leute auf und leben in Ehren. Wende Du Dich, Bertran, auch an ihre Güte!c، Später aber fand Augier Grund, bitter über den Geiz der Grolsen und über seine Stellung zu klagen (No. I, III). Die beiden Sirventese (No. I und No. 2) erzählen uns, dafs er viele

1 Vgl. Diez, Altrom. Sprachd. 124; Jahrb. 14, 292. 119; Ztschr. 2, 198. 9, 120; Romania X, 263.

$4^{*}$ 
Barone kennen lernte, die geizig waren, die das Dichten verachteten und so ungebildet waren, dafs Augier sich selbst mit seinem geringen Wissen im Vergleich zu ihnen erhaben fühlte. Diese ungebildeten Reichen hatten ihn so darben lassen, dafs er aller Habe entblölst war. Besonders schlecht erging es ihm, als er sich zu einer Reise verleiten liefs, um seine Heimat, das Viennois, und seine Eltern wiederzusehen. Drei Jahre hielt ihn eine thörichte Leidenschaft (un fols destrics) auf in diesem Lande, wo „das Geben Schmerz zu sein scheint".

Als rühmliche Ausnahmen, als Freunde der Dichter und als Augiers Freunde im besondern, nennt das Sirventes No. I den Markgrafen von Montferrat (Bonifaz II.), den Raimon Berengar, womit jedenfalls der Graf Raimon Berengar V. von Provence gemeint ist, und den Guigo Guiz Alamanz. Letzterer gehört nach Schultz der in dem Delphinat verbreiteten Familie Alamans an und wird als Guigo Alamans in Urkunden erwähnt ${ }^{1}$ (1201-2).

Dieses Hauptsirventes (No. I) ist nach dem 22. November 1220 , an welchem Tage Friedrich II. zum Kaiser gekrönt wuirde, verfalst; genauer läfst sich die Zeit nicht bestimmen. Die Vergleichung des Inhaltes von No. I und No. 9 macht es aber wahrscheinlich, dafs die drei schlimmen Jahre im Viennois später liegen als No. 9, das nach unserer obigen Annahme nicht lange vor 1225 fällt. Somit erhielten wir für No. I etwa das Jahr 1230 als Entstehungszeit.

Die Canzone No. 7 fällt später, sie ist überhaupt das späteste Gedicht Augiers, das wir bestimmen können. Sie nennt in der Tornada den Kaiser, „welchem Geben gefällt und höflich erscheint“. Also ist Augier noch einmal mit ihm, den er schon als König vor 1212 bewunderte, zusammengekommen, und Friedrich ist als Kaiser noch so freigebig wie einst. Wollten wir das Lombardia der Lebensbeschreibung streng als Oberitalien fassen, so liefse sich die Zeit des Gedichtes ungefähr festlegen. Es mülste dann im Frühling 1226, als Friedrich in Oberitalien Vorbereitungen zum Kreuzzuge traf, verfafst sein, oder es fiele erst nach $123 \mathrm{I}$. Letzteres ist nach dem über No. I Gesagten das Wahrscheinlichere.

Es erübrigt noch, die Tenzone No. $8 \mathrm{zu}$ betrachten. Genau wissen wir weder Zeit noch Ort. Das Urteil spricht darin ein gewisser Romieus, der reich und gebildet zugleich ist. Man könnte hier an den Kanzler Romieus des Grafen Raimund Berengar denken. ${ }^{2}$ $\mathrm{Da}$ Augier, wie oben erwähnt, bei Raimund Berengar Gastfreundschaft genofs, so liegt die Annahme nahe.

Der Inhalt der Tenzone zeigt den armen Joglar, der sich Reichtum wünscht, der den Reichtum schätzen lernte, als ihm Bildung nichts half.

Mehr wissen wir von Augiers Leben nicht. So gut nun auch Augiers Auswanderung nach Italien durch den Tod seines Gönners

1 S. Ruffi, Hist. de Prov. 140; Chevalier, Docum. inéd. rélat. au Dauphiné II 24 im Cartul. de l'église de Die (Schultz).

a S. Hist. litter. t. 20 p. 592. 
und den Albigenserkrieg begründet und die Identifikation der beiden von Bartsch getrennten Dichter gerechtfertigt scheint, so fällt doch immer noch auf, dafs die drei sicher in oder bei Béziers entstandenen Lieder (No. 3, 4, 5) den Zunamen Novella, die sicher nach 1220, also nach des Dichters Auswanderung nach Italien und wahrscheinlich in diesem Lande entstandenen (No. 1, 2, 7) den Vornamen Guilhem vermeiden und dafs nur das eine in zehn Hss. überlieferte Lied No. 6 (Ses alegrage) die Namen Augier Novella und Guillem Augier neben einander zeigt. Wollen wir einen Grund hierfür finden, so müssen wir annehmen, dals Augier zwar den Vornamen Guilhem hatte, dals sich aber in den zehn Jahren, welche ja wenigstens zwischen Lied No. 5 und Lied No. I liegen, im fremden Lande der Name verändert, der Schwerpunkt verschoben hat, vielleicht weil damals sehr viele Joglars den Vornamen Guilhem führten (s. Chabaneaus Reg. unter "Guilhem"). Das grolse Descort No. 6 aber scheint über Oberitalien hinaus auch in Gegenden bekannt geworden zu sein, wo man den Dichter noch bei dem alten Namen kannte. Vielleicht verdankte es diese weite Verbreitung seiner Beliebtheit, worauf die grofse Anzahl (10) der erhaltenen Handschriften deutet. Vielleicht brachte Augier es selbst mit in die Provence; denn die drei Jahre in der Heimat fallen ja wohl wenig später. Sollte es damit zusammenhängen, dafs die Hss. $C$ und $R$, welche allein den Namen des Dichters in der alten Gestalt angeben, den Vers 104, worin die italienische Geliebte angeredet wird, mifsverstehen oder absichtlich ändern?

Nichts hat unser Joglar Guilhem Augier zu thun mit einem um die Mitte des 13. Jahrh, lebenden Guillem Augier, der ein vornehmer, reicher Mann und ein Gönner der Dichter war. ${ }^{1}$

Auch die in den Cartulaires inéd. de la Saintonge p. p. Th. Grassilier, Niort 187 I öfter (z.B. S.65, 89, 106, 165) genannten Träger des Namens Guillem Augier lassen keine Beziehung zu unserm Dichter erkennen.

Nach dem Gesagten erfährt das Verzeichnis der Dichter bei Bartsch folgende Aenderungen:

14) Albert Bernart de Durfort.2

1) E'ra quan l'iverns nos laissa, Gauberz en Bernart de Durfort D, Ogiers IK.

2) Gausbert, razon ai adrecha $\mathrm{CD}^{\mathrm{a}} \mathrm{EG}(\mathrm{R})$, Tenzone. Arch. $35,102 \mathrm{G}$.

88) Bertran de Preissac. ${ }^{2}$

I) Era quan plor e iverna D, Gavauda C, Albert Cailla IKd. MG. 752-53 CI.

2) Gausbert, razon ai adrecha S. 14, 2. Tenzone zwischen Albert und Bertran.

1 Vgl. O. Schultz in der Ztschr. f. rom. Phil. IX II9 u. 120.

a Vl. ebd. VII 181 . 
60) (Bernart de Durfort) fällt weg.

37) (Augier Novella) fällt weg.

42) (Auzer Figueira) fällt weg.

205) Guillem Augier Novella.

1) Bertran, vos qu'anar solialz ab lairos E. Tenzone. MG. 535. (No. 9).

2) Cascus plor e plaing son dampnatge, Guillem Mogier de Beziers C, Augier R. MG. 579 R. R. 4, 46, Azaïs 122 unter Guillaúme de Béziers. (No. 3).

3) Erransa CR wie 2. R. 3, 133. Galv. 107. Azaïs 126 wie 2. (No. 4).

4) Guillem, prims iest en trobar a ma guia EMR(a). LB. 94 R. Chr. 67 R. (No. 8).

5) Laig faill cors e sabers e senz, Ugiers de Sant Donat F. Stengel, Marb. Univ.-Progr. 1878 No. 176. (No. 2).

6) Per vos bela douss'amia, Ogiers D, Ogier Niella C, Ugiers de Viena F, Uc de S. Circ E. R. 3, 104. PO. 397. (No. 7).

7) Quan vei lo dous temps venir, Guillem Augier $\mathrm{R}^{2}$, Guiraut de Borneil CR. MG. 863 R. (No. 5).

8) Ses alegratge, Guillem Augier R, Ogiers IK, Ogiers Novella D, Augier de Sant Donat C, Guillem Mogier C reg., Guiraut de Calanso C reg., Peire Raimon Sc, anonym MNW. MG. $285 \mathrm{~N}, 580-583$ CMRS. (No. 6).

9) Tos temps serai sirvens per deservir, Ogier ADHIK, Arnald Daniel c, MG. 577 I. Monaci, Studi III No.609 A und V No. 127 H. (No. 1).

\section{Die Gedichte.}

No. 1 .

Ueberlieferung.

Das Sirventes Toz temps ist in sechs Hss. überliefert, von denen zwei, I und $\mathrm{K}$, völlig übereinstimmen. Das Abhängigkeitsverhältnis der sechs Hss. ADHIKc wird durch folgende Figur veranschaulicht:

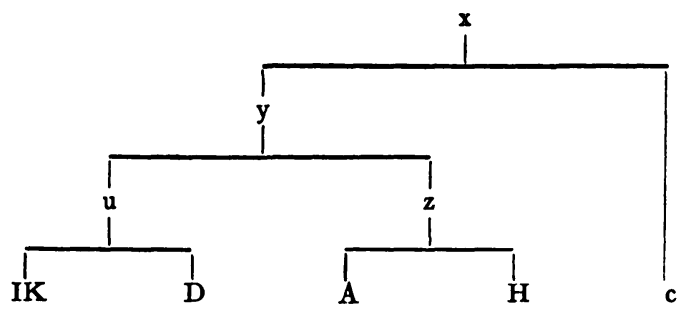
Fehler:

Dals IKD enger zusammengehören, beweisen die gemeinsamen

I 2: en sirven statt en sirventes, wodurch der Vers eine Silbe zu wenig erhält. 


\section{4: conseillador statt conseilladors.}

IV 25: so fehlt, deshalb hat der Vers eine Silbe zu wenig.

$\mathrm{Dafs} \mathrm{A}$ und $\mathrm{H}$ enger verwandt sind, geht aus dem gemeinsiamen Fehler II 12 escosalz statt escorsatz hervor, abgesehen von anderen Gemeinsamkeiten im Ausdruck. Auch fehlt in A und $\mathrm{H}$ die Tornada II.

$\mathrm{u}$ und $z$ stehen als Gruppe y der Hs. c gegenüber; denn e:inerseits bleibt in allen Hss. der Gruppe y Vers I 6 dunkel, der erst durch $c$ richtig gestellt wird, andererseits zeigt c starke Abweichungen im Ausdruck gegen alle Hss. aus y. (S. die Varianten.)

\section{Sirventes. (ADHIKC).}

(Orthographie von $D$.)

I.

Toz temps serai sirvenz per deservir

En serventes als flacs rics, d'aver sers.

Quar de lor vei consseilliers e convers

Conseilladors, que fan aunor aunir;

5 Per qu'en lor corz cortas d'enseignamen

Non a sol sen ni cab hom enseingnaz.

Neis eu mezeis, que no sui trop apres

$\mathrm{Ni}$ trop prezaz, quan mi pren, mi tenc pres.

II.

Mas vianar volgra de Vianes,

10 Qu'anc piez non fo viananz aviaz

Qu'eu fui, quan vinc ves mos parenz paraz.

Pero baros corz, escorzaz cortes

Ai trobat mainz, on non val ren trobars.

Tan son valen que val. Tant m'an valgut,

15 Qu'anc piez no fo ros a Roma romieus,

Que'm son del meu, desamaz entre Is mieus.

III.

Sapchan, se'm des lo rics donaire dieus

Poder, li ric despoderat perdut

2 En $A D H I K$, De $c$; seruentes $A H c$, seruen $D I K$; als $A D H$, al $I K c$; flacs $A D I K c$, crois $H-3 \mathrm{Qar} A D J K C$, Qe $H$; de lor vei $A D H I K$, uei de lor $c$; conseilliers $A D H I K$, conseiller $c-4$ Conseilladors $A H c$, conseillador $D I K$; aunor $D H I K c$, bon pretz $A-5$ Per qen $A D I K$, E de $H_{1}$ Et en $c$; cortas $A D H I K, \ldots$ as $c-6$ sol sen ni] sol senz ni $c$, sol sen nei $H$, sol seing ni $A$, fol seigni $D$, sol seignei $I K$; Non a $A D I K$, Noia $c$, Caill noi $H-7$ Neis $A D I K c$, Per $H$; eu $A c$, qeu $D H$, qeus $I K-$ 9 uolgra $A H I K c$, uolgar $D-$ Io piez $D H I K C$, pretz $A$; fo $A H I K$, fehlt $D c$ - I Q Qeu tui $A D I K c$, Cum eu $H$ - I2 Pero $D H I K c$, Per los $A$; escorzaz $A D K$, escosatz $H c$, escortatz $I-$ I 3 Ai trobat $D H I K c$, Ab trobars $A-14$ Tan $D H I K$, Qieu $A$, Qen $c-15$ piez $H c$, miellz $A D I K$; ros $A D H c$, ni ros $I K$; romiens $A D H K c$, mnieus $I-16$ Qem son $D I K$, Qio fui $A c$, Qeu soi $H$; desamaz $D I K$, desarmatz $A$, desmaratz $H$, desaimaiz $c-17$ Sapchan sem des lo rics $A D I K c$, Aissim done lo rics $H$; donaire dieus $A D H I K$, dona i rei dons $c-18$ li ric $A I K$, quill ric $H$, del rics $c$; despoderat $D H I K$, despoderatz $c$, desesperat $A$ 
Qu'eu son aitals, s'ieu quezes lor ajut,

20 Com fon Judas que-l vendet als Juzeus.

Lor frech frach fag m'an lag desafaitat

$E$ desolat de solaz e de chan.

Lor arriër son orriër de jai

Vil, de vilan charau escaravai.

IV.

25 A, quan mal viu qui ve so que ill desplai, Que-I desplazer sai ve que ill croi ric fan!

Qu'eu n'ai vist mainz cui prez trai de soan,

Que soanan puois prez, quan ben lor vai.

Qu'eu vi ja.l ric rei Rogier Frederic

30 Fresc ses esfrei per valer a valor:

Ja non cugei, tan l'auzi prez prezar, Que ja.l pogues emperis pejurar.

$\mathrm{V}$.

E Monferat se solon referar

$\mathrm{Li}$ desferat, valen al valedor.

35 Per so no i a desenseignat seignor, $\mathrm{Si}$ tot non fai loing sa valor volar.

Mas eu com fols volei a mon voler

En Vianes, on par dolors donars,

On fols destrics m'a trebaillat tres ans,

40 Qu'anc plus estraing trebaill non trais truanz.

A n Raimon rest Berenguier prez prezanz,

Cui non par dars dolors ni redars danz.

Per son païs dels adreiz trespassanz

Es Guiguo Guiz mos amics Alamanz.

19 Qeu son aitals $I K$, Qe son aital $A D$, Fosson aital $H$, Aital fossio $c$; sieu qezes $A c$, qi qezes $D$, qi crezes $H$, si qezes $I K-20$ qel $A D I K c$, qels $H$; fo $A D H I K$, fu $c-21$ Lor frech frach fag $D I K$, lor freit faich mant $A$, li sei croi fait $H$, lor faitz fals fraich $c$; man lag desafaitat $D H I K$, laichamen desafiat $A$, man laitz desafetait $c-22$ solaz $A D I K c$, dompnei $H-23$ Lor arrier son orrier de iai $D I K$, Lor orrier son orrier de iai $A$, Lor oreder son oreder de gai $H$, Lor eresier so non rosat de gai $c-$ 24 charan escarauai $A D I K$, garent esçarauai $H$, cheranz escheruai $c-$ 25 qan $D H I K c$, cum $A$; ve] fehlt $I$; qeill $A$, qe lle $D I K$, qel $c$, qille $H$; so $A c$, fehlt $D H I K-26 \mathrm{Qi}$ uell croi fait qill auol baro fan $H$; sai ue $A D I K$, son frutz $c-27$ Qeu $A D H c$, Com $I K$; nai uist $A D H$, en ue $I K c$; cui $A D H I K$, au $c-29$ rei fehlt $H-30$ Franc $D$, Feran $H$, Fresc $A I K c$; a ualor $A D H I K$, auolar $c-31 \mathrm{Ja} D I K$, E $A$, Ni $H$, Qan $c-$ 32 emperis $A D I K$, emperi $H$, lemperes $c-33$ solon $D H I K c$, solion $A-$ $34 \mathrm{Li}$ plus ualen ab ualen ualedor $H-35$ Per so $D I K$, Pero $A H$, Per $c$; desenseignat $A H$, deseignat $I K$, deseinseigna $D$, dels ensenhatz $c-36 \mathrm{Si}$ tot $A D I K$, Si pot $H$, Se tuit $c$; fai $A D H I K$, fan $c-37$ Mas $A D I K c$, Et $H-38$ par $D H I K c$, pars $A-39$ fols $A D H$, fuilh $c$, ros $I K$; destrics $A D I K$, trebalh $c$, dezirs $H$; tres $H I K c$, IIII $A D-40$ non $A D H c$, noi $K$, nois $I$; trais $A D I K$, trai $H$, trac $c-4 I$ fehlt $c$; An $D H I K$, A $A$; rest $H$, ret $D I K$, uai $A-42$ fehlt $c$; redars $A D H$, te dars $I K-43$ fehlt $A H c-44$ fehlt $A H c$; Guigo $I K$, gigi $D$. 
Anmerkungen.

I 6. Millot (Hist. litt. des Troub. I 342) übersetzt: „Personne ne peut indiquer par un signe de tête un homme bien appris". Ich lese mit c, senz in sen wandelnd: non a sol sen ni cap hom ensenhats.

II 12. Raynouard nimmt Lesart I escortatz d. h. „écourté = gekürzt, gestutzt"; besser ist offenbar die Lesart von $\mathrm{K}$ und c: escorcatz $=$,geschunden", was an unser "schäbig" erinnern würde.

III 23. Der Vers scheint rettungslos entstellt. Mistral führt unter gai (jai) die Redewendung auf un orre jai $=$,un vilain moineau, homme"; doch ist damit der Stelle nicht aufgeholfen, solange die dreisilbigen Wörter arriër, orriër (Var. oreders) unerklärt bleiben.

III 24. charau ist eine einfache und notwendige Aenderung aus dem charan der Handschriften. Vgl. Mistral unter carrau. Also „Landstrafsenkäfer", wobei wir wohl an die Kotkäfer der Landstrafse zu denken haben.

Metrisches.

Das Lied ist ein Sirventes (vergl. V. 2). Es besteht aus fünf Coblas und zwei Tornadas. Die Coblas sind Coblas capcaudadas (vergl. Leys d'amors p. p. Gatien-Arnould, I 84 I, I p. 236) von je acht Zehnsilblern mit Cäsur nach der vierten Silbe, und jede Tornada hat zwei Verse. Der Versausgang ist ebenso wie die Cäsur immer männlich. Das Reimschema ist:

$$
\begin{aligned}
& \text { I. Str.: } a \text { b b a } \mathbf{x} \text { c d } d \\
& \text { 2. Str.: d c c d } \boldsymbol{y} \text { e f } f \\
& \text { : } \\
& \text { 5. Str.: } \mathrm{k} \text { i i } \mathrm{k} \text { = } 1 \mathrm{~m} \mathrm{~m} \text { (Torn. } \mathrm{m} \mathrm{m} \text { ). }
\end{aligned}
$$

In jeder Cobla findet sich ein Korn, das erst in der nächsten Cobla reimt. Drum bleibt 1 in der letzten Cobla ohne Reim. Aufserdem begegnet in jeder Strophe ein reimloser Vers (oben $x, y, z$ bezeichnet). So haben wir im ganzen sechs Verse, die nicht reimen.

$\mathrm{Da}$ reimlose Verse an sich schon sehr selten in der provenz. Poesie vorkommen, so ist es nicht wunderbar, dafs wir sonst keine Strophe desselben Baues finden.

Die Strophe ist unteilbar.

No. 2.

Sirventes avols e descortz. $(F)$.

Laig faill cors e sabers e senz

Als malvatz rics homes avars, Qui nejon que metres e dars Es valors e tẹners niënz.

5 Que d'aquel es l'aurs e l'argens Que'n fai dos e cortz e guerra, Non ges d'aquel que'l soterra. Qu'ab dar fo Alixandres rics E Daires per tener mendics.

Io Dels malvatz baros recrezenz, A cui dos e cortz par guerra, 
Sui sers, quant hom los soterra. Quar tan m'es lor vida destrics, Per qu'a la mort lor sui amics.

Avers engana'ls malvatz rics

E.ls pros creis d'onor e d'amics.

\title{
Anmerkungen.
}

Das Gedicht ist nur in Hs. F erhalten. Ich fand nötig, das uezon in Vers 3, welches Stengel (F) druckt, zu ändern in nejon, also: „den Geizigen,, welche leugnen, dafs Spenden und Geben Tugend ist ....".

In Vers 2 wurde homs der Hs. in homes geändert.

\section{Metrisches.}

Die Hs. F, welche allein das Gedicht Augiers überliefert, nennt es ein Sirventes avols e descortz; das würde also ein "schlechtes Sirventes-Descort" sein. Mit Rücksicht auf die Metrik verdient es die Bezeichnung „Descort“ nicht; denn auf die übermärsige Grölse der Tornaden (sieben Verse) im Verhältnis zu der einzigen Cobla von neun Versen dürfte der Ausdruck doch kaum abzielen. Auch in dem Liede des Bonifaci Calvo Un nou sirventes ses tardar, welches Mario Pelaez un sirventese-discordo nennt (Genova I89 I), bietet die Metrik nichts Auffälliges, da es aus vier siebenzeiligen Strophen von je sieben männlichen Achtsilblern besteht. Der Ausdruck mufs sich also auf den Inhalt beziehen. Sicherlich liefsen sich dann aber sehr viele Sirventesen als Sirventes-Descorts bezeichnen. In unserem Falle läge übrigens eine Aenderung von descortz in descortes recht nahe, da das synonyme Adjektiv avols daneben steht.

Das Lied besteht aus einer Cobla von neun Versen und zwei Tornaden von fünf und zwei Versen. Formel:

$$
8 \mathrm{a} 8 \mathrm{~b} \mid 8 \mathrm{~b} 8 \mathrm{a} \| 8 \mathrm{a} 7 \mathrm{c} \cup 7 \mathrm{c} \cup 8 \mathrm{~d} 8 \mathrm{~d}
$$

Teilbar in zwei Pedes und eine Syrma. Diesis nach dem 4. Vers.

\author{
No. 3 . \\ Planh. $(C R)$.
}

(Orthographie von $C$.)

I.

Quascus plor e planh son dampnatge,

Sa malenansæ sa dolor;

Mas yeu, las! n'ai e mon coratge

Tan gran iræ tan gran tristor,

5 Que ja mos jorns planh ni plorat

Non aurai lo valent prezat,

Lo pro vescomte, que mortz es,

De Bezers, l'ardit e.l cortes,

3 nai $C$. ai ne $R$; Mas $C$, Et $R-7$ pro $C$, pros $R$ 
Lo gay e.l mielhs adreg ell blon

10 Lo mellor cavallier del mon.

II.

Mort l'an, et anc tan gran otrage

No vi hom ni $\tan$ gran error

Fach mai ni tan gran estranhatge

De dieu et a nostre senhor,

15 Cum an fag li can renegat

Del fals linhatge de Pilat

Que l'an mort; e pus dieus mort pres

Per nos a salvar, semblans es

De lui qu'es passatz al sieu pon

20 Per los sieus estorser, l'aon.

III.

Mil cavalhier de gran linhatge

E mil dompnas de gran valor

Iran per la sua mort arratge,

Mil borzes e mil servidor,

25 Que totz foran gent heretat,

S'el visques, e ric et honrat.

Ar es mortz! Ai dieus, quals dans es!

Gardatz quals etz ni quo us es pres,

$\mathrm{Ni}$ selhs qui l'an mort, cui ni don,

30 Qu'eras no us acuelh ni'us respon.

IV.

A senhor! ta fort deu salvatge

Esser al gran et al menor,

Quant del sieu honrat senhoratge

Nos membrara e de l'honor

35 Que'ns fetz e de la fezautat

Quan per nos l'agro mort jutjat.

Er es mortz, ai dieus! quals dans es,

Caitiu, cum em tug a mal mes!

Ves qual part tenrem, ni ves on

40 Penrem port? Tot lo cor m'en fon.

V.

Ric cavalier, ric de linhatge,

Ric per erguelh, ric per valor,

9 Lo $C$, El $R$; el mielhs adreg $R$, ladreg lo nou $C$; blon $C$, bon $R$ - ro Lo $C$, El $R$ - il Mort lan $C$, Mortz es $R$ - 13 Fach mai $C$, Mais far $R-14$ et a $C$, on sa $R-16$ del $C$, dels $R-18$ Pels sieus estorser lai ont es $C-19$ De $R$, A $C-20$ laon $R$, la on $C-$ III und IV sind in $R$ vertauscht -25 foran gent $C$, eran ben $R-26$ Silh uisques $C$, Siel uisquet $R-27$ quals $C$, tals $R-28$ Gardatz quals etz $C$, Guaratz qui em $R$; quous $C$, coms $R-29$ cui $R$, qui $C-30$ Queras $C$, Car us $R$; nous $C$, nons $R$; nius $C$, nins $R-34$ honor $C$, amor $R-$ 35 Quens $C$, Que $R$; e $C$, ni $R-36$ Vas selhs querona mort iutiat $R-$ $3^{8} \mathrm{em} C$, es $R-4^{0}$ penrem $C$, penre $R-4^{1}$ ric de $C$, de gran $R$ 
Ric de sen, ric per vassallatge,

Ric per dar e bon servidor,

45 Ric d'orguelh, ric d'umilitat,

Ric de sen e ric de foudat,

Belhs e bos, complitz de totz bes,

Anc no fo nulbs hom que'us valgues.

Perdut avem en vos la fon

50 Don tug veniam jauzion.

Selh dieu prec qui fetz trinitat

De se mezeis en deïtat

Qu'ell cel, on lo major gaugz es,

Meta l'arma, e non li pes,

55 Et a totz selhs qui pregatz son

De son ben socorre et aon.

Belhs papaguais, anc tan vezat

No'm tenc amors, c'ar plus torbat

No'm tenga $e \cdot l$ dan que ai pres

60 Del melhor senhor c'anc nasques

Aitan can clau mar en redon,

Que m'an mort trachor, no sai don.

43 de sen $C$, per sen $R-46$ ric de $C$, ric a $R-50$ veniam $C$, partiam $R-52$ De se que en lo loc onrat $R-53$ Sus el $R-54$ larma $C$, la son arm $R$; non li $C$, nol $R-55$ a totz $C$, totz $R$; son $C$, en son $R-$ 56 Dels sieus bes $R-60$ canc nasques $C$, com agues $R-{ }^{8} 8-62$ ist in $C$ verstümmelt: nom .... is torbat $-\mathrm{mi} \ldots$ i pres - del $\mathrm{m} \ldots$ nc nasques $-a \ldots$ ars en redon $-q \ldots$ achors no sa...

\section{Anmerkungen.}

Azaïs stellt Torn. II zwischen Str. IV und V.

Auffällig ist die Wiederholung von ric per erguelh V 42 in Vers 45 derselben Strophe in der Form: ric d'orguelh und die des Reimwortes linhatge III $2 I$ und $V 4 I$.

II 20 l'aon $=3$. p. conj. praes. von aondar. Subjekt dazu wird am besten aus dem vorangehenden Satze ergänzt (= Christus).

\section{Metrisches.}

Das Lied ist ein Planh, ein Klagelied auf den ermordeten Gönner. Die erforderliche Feierlichkeit in der Form ist erreicht durch die gleiche Länge der Verse und die Gleichreimigkeit aller Strophen. Auch finden sich in jeder Strophe nur zwei Verse auf weiblichen Reim.

Das Lied besteht aus fünf Coblas unissonas von je zehn und zwei Geleiten von je sechs Achtsilblern.

Schema: $\quad 8 \bar{a} \sim \mathrm{b} a \sim b c c d d e$ e. 


\author{
No. 4 . \\ Descort. $(C R)$. \\ (Orthographie von $C$.)
}

Erransa,

Pezansa

Me destrenh $e^{\cdot m}$ balansa.

Res no sai, on me lansa

5

Esmansa.

Semblansa

Me tolh iræ m'enansa,

$E \cdot m$ dona alegransa

Un messatgier que me venc l'autre dia,

Io Tot en vellan mon veray cor emblar,

Et anc de pueys no fuy ses gelozia

$E$ res no sai vas hon lo m'an sercar.

Cum fis amaire

Murrai, ses cor vaire,

20

Ab sol que'l sien laire

No'm sia fals ni var.

Qu'aissi deu faire

Tot drut de bon aire,

Per que m'es vejaire

Que ben o deya far.

Per merce'us prec, bella dousset' amia,

$\mathrm{Si}$, cum ye'us am, vos me vulhatz amar.

Car ye'us am mais que nulha res que sia

Et anc no'us vi, mas auzit n'ai parlar.

25

30

Als no sai dire,

Mas dat m'avetz cossire

Tal don planc e sospire.

No puesc esser jauzire

De vos qu'ieu pus dezire, Tro veya rire

Vos don yeu suy servire.

Aculhetz me, no.us tire!

Quar trop sai del dezire,

Que crey que'm vol aucire.

3 em $R$, fehlt $C-9$ que $R$, fehlt $C-1$ I de pueys $C$, pueissas $R$ - 16 var] vas $C$, vars $R-17$ deu] o deu $C R-20$ deya $C$, deu $R-$ 24 mas $C$, ni $R-30$ veya $C$, sō car $R-31$ A vos $R$.

Anmerkung.

Von Azaïs weiche ich ab in Vers 17, wo er das $o$ der beiden Hss. beibehält (s. Metrik). Auch lälst er Vers 29 irrtïmlich aus.

\section{Metrisches.}

Dies Lied ist ein Descort, wenigstens wird man es am besten so bezeichnen, so sehr es auch an Tanzlieder u. a. erinnern mag. 
Strophen hat es nicht, man kann fünf strophenartige Abschnitte unterscheiden, an die sich der Sinn nicht kehrt. So greift der Satz vom ersten Abschnitt zum zweiten über (6a 10 ob). Dem Sinne nach ist es ein Descort (Eifersucht).

Reimschema:

$$
\begin{aligned}
& \left.\begin{array}{l}
(1-4) \quad 2 a \smile 2 a \cup 6 a \cup 6 a \cup \\
(5-8) \quad 2 a \cup 2 a \cup 6 a \cup 6 a \cup
\end{array}\right\} \\
& \text { (9-12) lobu 10c 1oburoc } \\
& (13-16) \quad 4 \mathrm{e} \smile 5 \mathrm{e} \cup 5 \mathrm{e} \cup 6 \mathrm{c} \\
& (17-20) \quad 4 \mathrm{e} \cup 5 \mathrm{e} \cup 5 \mathrm{e} \cup 6 \mathrm{c}\} \\
& (2 \mathrm{I}-24) \text { rob } \operatorname{loc} \text { rob } \cup \text { IOc } \\
& (25-29) \quad 4 d \cup 6 d \cup 6 d \cup 6 d \cup 6 d \smile \\
& (30-34) \quad 4 d \cup 6 d \cup 6 d \cup 6 d \cup 6 d \cup\}
\end{aligned}
$$

$\mathrm{C}$ und $\mathrm{R}$ geben Vers 17 beide fünfsilbig qu'aissi o deu faire: der Symmetrie mit Vers I 3 wegen (cum fis amaire) habe ich den Vers 17 durch Streichung des 0 auch viersilbig gemacht. Doch wäre aissi o mit einsilbigem io nicht undenkbar. Leicht liefse sich auch Vers $\mathrm{I} 3$ zu $5 \mathrm{e} \smile$ ergänzen (cum fizels amaire). Jedenfalls scheint Aenderung nötig, da das Lied sonst ganz symmetrisch gebaut ist.

\section{No. 5 . \\ Ueberlieferung.}

Das Descort Quan vey ist in drei Fassungen überliefert, nämlich zweimal in Hs. $\mathrm{R}$ und einmal in C. Ich bezeichne die beiden Fassungen in $R$ als $R^{1}$ und $R^{2}$.

$\mathrm{R}^{2}$ überliefert das Gedicht in Gestalt von 52 Versen und zwei Geleiten von je 4 Versen, also zusammen 60 Versen. $\mathrm{CR}^{1}$ geben Geleit I nicht und Geleit II nur zur Hälfte, also je 54 Verse.

Dafs $C$ und $R^{1}$ enger zusammengehören, ergiebt sich einerseits aus der fast ganz gleichen Ausdrucksweise, von welcher $R^{2}$ sehr häufig allein abweicht, andrerseits aus folgenden gemeinsamen Fehlern:

V. 25. $\mathrm{CR}^{1}$ haben: e mir m'en sas belas faisos, was I) nicht mit dem vorausgehenden Präter. laissiei harmoniert, 2) wenig Sinn giebt (,spiegele mich in ihren Zügen“).

V. 26. $\mathrm{CR}^{1}$ : el luec del cors $\left(\mathrm{R}^{2}\right.$ giebt richtig cor).

V. 28. CR 1 : no fui neis de leis poderos: ist sinnlos.

$\mathrm{R}^{2}$ hat in allen diesen Fällen das Richtige, $\mathrm{R}^{2}$ steht also der Gruppe $\mathrm{CR}^{1}$ selbständig gegenüber.

Stammbaum der Handschriften:

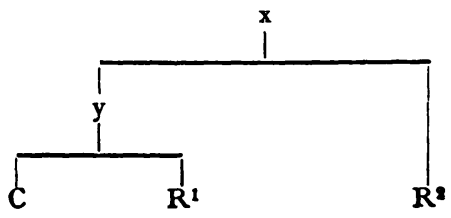


Descort. $\left(C R^{1} R^{2}\right)$.

(Orthographie nach $C$, in den Geleiten nach $R^{2}$.)

Quan vey lo dos temps venir

E la conhdeta sazon,

Que fai pratz reverdezir,

E floreisson li boisson,

Et aug los bois retendir

$\mathrm{Pe} \cdot 1$ chant que fan l'auzelon

E tot, quant es, esbaudir

Per amor o pauc o pron,

Mas ieu, qui suelh estar en alegrier

Io E mains d'autres de mon joi alegrar,

Planh e sospir e muer de dezirier

E plor dels huels ab que vill dous esgar,

Que'm fes selha qu'era'm torn'en niën,

Al prim qu'ieu vi sa gaya captenensa;

$15 E$ fai o mal merces, quar no dessen

En liei que a totz jorns melhur'e gensa.

Qu'aitan destreg la prejon mei voler;

$E$ pos no.lh falh nulhs bons aips mas merces,

Merces pogra tot lo grat retener,

20 Sol que'l sieu cors plazent metre's volgues,

Franc, d'aut paratge,

Sens mal uzatge,

Tant que coratge

Part l'omenatge

25 Laissiei en sa francha bailia

$\mathrm{E}$ mis m'en sas bellas faissos

En luec del cor, si qu'anc pueis dia

No fui de mi eys poderos.

Mas quan la vey, li cug ab los huels dire

30 So que no l'aus ab la boca retraire.

Mas empero nulha tan no dezire

Ni contra lieys nulh'autra no pretz guaire.

Doncs qual esfors fai, se vas mi s'aiura,

Ma bona dona, ni.m torna en viutat?

$3 \mathrm{E}$ vey los pratz reuerdir $R^{2}-5$ lo boi $C R^{1}$, los bois $R^{2}-6$ que $C R^{2}$, quei $R^{1}-8$ o pauc $C$, e pauc $R^{1} R^{2}$ - $9 \mathrm{Et} R^{2}$ - 10 de mon $C R^{1}$, pel mieu $R^{2}-$ II e muer de dezirier $C R^{1}$, fehlt $R^{2}-12$ uil $C R^{2}$, un $R^{1}$ - I3 queram $C R^{1}$, qiam $R^{2}-15$ no dessen $C R^{1}$, nom defen $R^{2}-16$ En liy $R^{1}$, A leys $R^{2} ; \overline{\mathrm{q}}$ a $R^{2}$, que $C$, ca $R^{1}$; melhur e gensa $R^{1} R^{2}$, melluj re gensa $C^{\prime}-17$ Pus tan planhen $R^{2}-18$ pos $C R^{1}$, car $R^{2}-19$ Merces $C R^{1}$, fehlt $R^{2}$; tot lo grat $C R^{1}$, totz sos gratz $R^{2}-20$ Ab que del sieu cors ualer mi uolgues $R^{2}-21$ Francx $R^{2}-22$ Fetz maluestatie $R^{2}-$ 23 Per quel cor o dia $R^{2}-24$ lomenatge $C R^{2}$, lo me natic $R^{1}-25$ sa $C$, la $R^{1} R^{\mathbf{2}}$; francha $C R^{1}$, vostra $R^{2}-26 \mathrm{E}$ mis m'en] $\mathrm{E}$ mirmen $C R^{1}$, Em mis en $R^{2}-27$ cor $R^{2}$, cors $C R^{1}$; que anc $C R^{1}$, que $R^{2}-28$ de mi eys $R^{2}$, neis de leys $C R^{1}-29$ Ans cã lesgar o cug $R^{2}-31$ Pero ben sap que res $R^{2}-34 \operatorname{nim} C R^{1}$, ni $R^{2}$ 
35 Que nulha res ni hom no s'en rancura.

Ben degr'aver d'eis lo sieu piëtat!

Qu'ieu non ai d'autr'esperansa

Que ja'm leuje ma dolor.

Gens cors ab humil semblansa,

40 Genser que roz'en pascor,

Per dieu! prendez m'acordansa,

Prometetz mi vostr'amor!

Que, si'm breu no'us pren pitansa,

Auciran mi planh e plor.

45 E ja del plus no'us prejarai

Qu'ans si'en la vostra merce:

Retenez me, que be'us eschai,

E conoissetz qu'autra ni me

Non am tan, per la fe qu'ieu dey

50 A la pros marqueza gaya

De Menerba, a cuy sopley,

Quar es sa valors veraya.

Vas n'Alazays, c'apres a drey,

De Boyssazo, que ben aya,

55 T'en vay, descort! c'om no la vey

Que de leys ben non retraya.

Bels papagays, si tot no'us vey,

Far podetz tot, quant vos playa,

De me; on que m'an ni m'estey,

60 Vostra doss'amor m'apaya.

$35 \mathrm{E}$ pus hom mays ni res nō $R^{2}-36$ sieu $C R^{2}$, sen $R^{1}$; pietat $C R^{1}$, domneyat $R^{2}-38$ jam $C R^{2}$, jāz $R^{1}-39-44 R^{2}$ ordnet: 43,44 , $39-42-39$ Gay $R^{2}-41$ prendetz macordansa $C R^{1}$, prend vos mēbrāsa $R^{2}-44$ Auciran $C R^{1}$, Ausir man $R^{2}-46$ Ans sien las vostras merces $R^{2}-47$ que $C R^{1}$, car $R^{2}$; eschai $C$, chai $R^{1}$, estay $R^{2}-48$ Pus $R^{2}-$ 49 qieu $C R^{1}$, qus $R^{2}$ - 51 a $R^{1}$, vos $R^{2}$, fehlt $C$; valors $C$, valor $R^{1} R^{2}-$ 53-56 fehlt $C R^{1}-58$ tot quant vos $R^{2}$, de mi queus $C R^{1}-59-60$ fehlt $C R^{1}$.

Anmerkungen.

Vers 33 s'aturar heifst 1) sich aufhalten, zögern - 2) sich anstrengen, streben (Stimming, Glossar zu Bertran de Born).

Vers 49-5I. Hier wurde die Lesart von $C^{\prime}$ bevorzugt, weil gegen das $\bar{q} u s$ und vos cui von $R^{2}$ das sa in Vers 52 spricht.

\section{Metrisches.}

Dies Gedicht ist ein Descort. Es lärst sich einteilen in fünfzehn Abschnitte von je vier Versen, Abschnitte, die aber keine Strophen sind. Denn der Satzbau kehrt sich nicht an die Grenze der Abschnitte. 


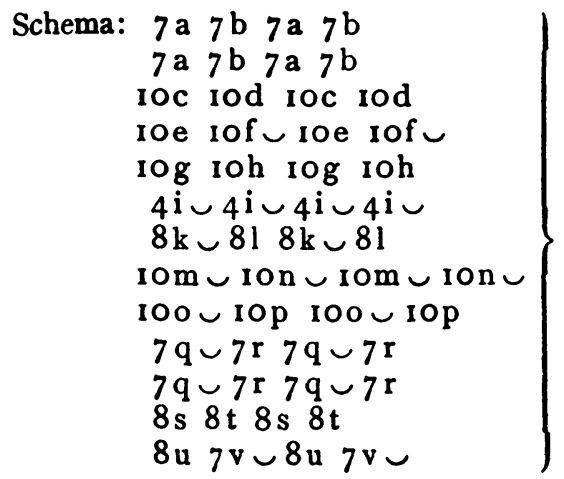

Also 52 Verse mit 21 verschiedenen Reimen. Die Zehnsilbler haben männliche oder lyrische Cäsur nach der vierten Silbe; V. 16 hat keine Cäsur; V. 34 hat weibliche Cäsur nach der fünften Silbe mit Verkürzung der zweiten Vershälfte $(5+5)$, wie sie häufig (z. B. bei Peire Vidal) vorkommt.

Dazu kommen noch zwei Geleite von je vier Versen, also im ganzen 6o Verse mit 21 Reimen.

\section{No. 6.}

\section{Ueberlieferung.}

Das Descort Ses alegrage ist dasjenige von Augiers Gedichten, das sich am häufigsten in den Hss. findet, nämlich in 1o Hss. Das Abhängigkeitsverhältnis zeigt folgendes Schema:

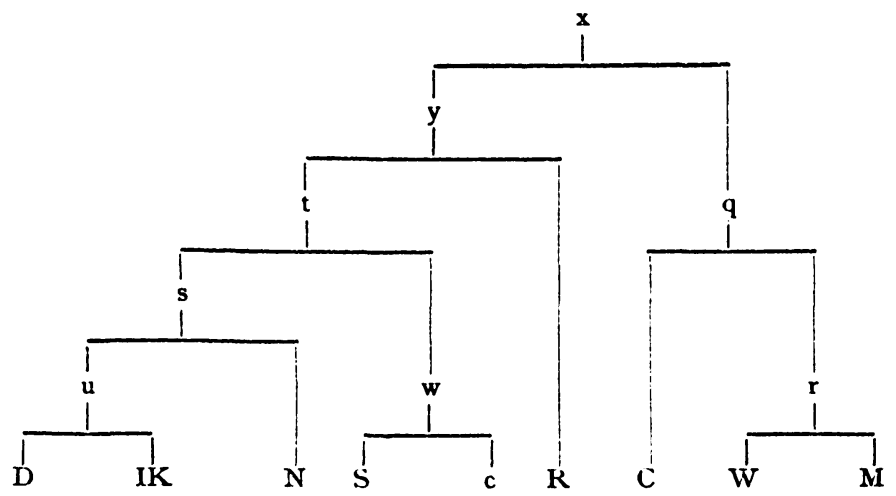

DIK, die schon oben im Sirventes Toz temps und auch sonst zusammengehen, zeigen hier ihre Verwandtschaft durch den ihnen eigenen Fehler: 12 maint meza (für malmę̧a).

Gruppe s charakterisiert sich durch Auslassen von Vers 45, den alle übrigen Gruppen aufweisen, aufserdem durch Gleichheit des 
Ausdrucks, z. B. voillats (33) und Doncs (15) gegen plasaos bezw. Mas der übrigen.

$S$ und $c$ zeigen sich nahe verwandt durch gleiche Ausdrucksweise (vergl. Varianten) und folgende gemeinschaftliche Fehler: 12 Mamia statt malmeģa - 22 tornet statt dobla - 28 deschaia statt estraia - 58 fehlt Sc.

Gruppe $t$ lärst sich nur aus Gleichheit der Ausdrucksweise, besonders der gleichen Auffassung des Verses IO4 erschlielsen (vergl. Varianten).

$R$ steht in Auffassung und Ausdruck meist selbständig für sich, aber doch immer $t$ näher als $q$, so z. B. V. 46 . Gemeinsame Fehler zwischen $t$ und $\mathrm{R}: 7$ reclus ni estatge (sinnlos) - 30 per statt part.

$M$ und $W$ gehören eng zusammen. Gemeinsame Fehler: 7 überflüssiges d'estage, was die Symmetrie der Str. I mit Str. II stört und auch keinen Sinn giebt - 77 quar statt quier 86 fehlt - 107-108 Reimworte Cortes: Es, wo das Schema Reim auf -en verlangt. Es ist aufserdem sinnlos.

Gruppe q charakterisiert sich durch einen gemeinsamen Fehler: 46 em conjura statt fes c'om jura und durch mehrere gleiche Ausdrücke, so z. B. 9 fo (y hat $a c)-87 E l$ (q hat $D e$ ) etc.

(Orthographie nach $M$.)

I.

Ses alegrage

Chant per agradage. Follage

Fatz, quar mon corage

5 S'es lai on s'es mes;

Qu'anc plus salvage

Reclus d'ermitage

Del mieu senhorage

Non fo nulls hom pres.

II.

10 Qu'aissi fos presa

Del mal que m'adeça, Malmeça

Cella, cui pauc peza

Car mi fai languir!

I5 Mas on er queza

Merces ni franqueça,

7 dermitage $C$, de boscage destage $M W$, ni estage $D I K S C$, ni ostatie $N R$ - 9 fo CMW, ac DIKNRSC - Io Car si $N R$ - II gem CDIKN $R S c W$, qan $M$ - 12 Malmeça $M W$, Maint mesa $D I K$, Ma meza $C N$, Mamera $R$, Mamia $S c-13$ fehlt $W-14$ languir $C D M N S c W$, murir $I K R$ - 15 Mas MRScW, Doncs DIKN, Dieus $C$ 
Pos la plus corteza

-M vol ses tort auçir?

III.

Per miells aucire

20 Mi tornet en jai

Del lonc cossire,

Pueis dobla'm l'esmai.

Soven m'albire

Que tot m'en partrai;

25 Pueis, quan me vire,

Ieu trueb mon cor lai.

IV.

S'er ja qu'ieu l'aia,

Greu er quie'm n'estraia

Sagramen ses paia

30 Per fals mot escur.

Ai, bella e gaia,

Plazenz, non veraia,

Plasa'os que'us desplaia

$\cdot \mathrm{L}$ grieus mals, don endur!

V.

35 Lonja endura

M'en agura

Aventura

Tal, si'm dura

Part mezura,

$40 \quad$ Que'm pejura

-L grieu mal que m'auci

$\mathrm{Si}$

Que drechura

Ni falsura

45

$\mathrm{Ni}$ segura

18 ses tort $C D M N S C W$, sos tortz $I K$, del tot $R$; aucir] aucire $R S-$ 19 miells $C D I K M S c W$. mi $N R$ - 20 jai $C D I K M N R c$, lai $S$, ay $W-$ 2 I Del $C M N R S C W$, Dun $D I K$; cossire] sospire $D-22$ doblam $C W$, dobla $D I K N$, doblet $M$, doblem $R$, tornet $S c-23$ malbire $C D M S c$, balbire $N$, marbire $I K W$, malbira $R-24$ Que tot $I K M W$, Que totz $D N S c$, Quades $C R$; partrai] partira $R-25$ quan $C D M N S c W$, sol $I K R$; vire] uira $R-27$ Senz za $N-28$ er $C D$, cug $I K N R$, cre $c$, mes $M W$, fehlt $S$; nestraia $C I K M N R W$, deschaia $S c$, entraia $D-29$ ses plaia $C N-$ 30 fals] foll $M W-33$ Plasaos $C M S i W$, Uoillaz $I K$, Uoillan $D$, Uillaz $N$, Sieus play $R$; qeus desplaia] qieu desplaia $M$, no dechaia $R-34$ Greus mals $C I K S W$, Greu mals $c$, Lo greu mal $N R$, Als grieus mals $M, L$ greus mals $D$; don] que $N R-36$ Non aura $S ;$ fehlt $N-37$ fehlt $D-38$ Tal seu dura $N$, fehlt $C-39$ fehlt $C N W$; Part $M$, Par $S c$, Per DIKR 39-40 von $M$ umgestellt - 40 Quem $M N W$, mi $C D I K R S c$ - 41 Greu mal IKMN, Lgreu mal $C D$, Lo mals $S c$, Greus mal $W$, Lo greu mal $R-$ $42 \mathrm{Si} C N S c W$, Sr $M$, fehlt $D I K R$ - 43 Que de rancura $c$ - $44 \mathrm{Ab}$ $M W-45 \mathrm{Ni}$ segura $C R S$, Mas segura $M$, fehlt $D I K N W c$ 
Fes c'om jura

No meilhura,

Anz s'atura.

Quan la prec, de mi

50 $\mathbf{R i}$.

VI.

Qu'anz volria,

Sol que mia

Fos un dia

En ma via,

55 Non daria

$\mathrm{Ma}$ folia

Per quatorze senz

Venz.

E cug, sia

60

Que m'enbria

Doncs fadia,

M'en partria,

S'ieu podia.

Mas so'm lia

65 Qu'om vencutz sufrenz

Venz.

VII.

Be's tainh vensa

Benvoilhensa

Lials, que non chai,

70

Lai

On semenza

De valenza

Floris ab verai

Jai.

75 Mantenenza

Ab temenza

-Us quier, donna, si'us plai,

Sai.

Que cell gensa

46 Fes com iura] Fes coniura $D R S$, Fos com iura $I K$, Em coniura $C M$, Ses giura $c$, Fes con cura $N$, fehlt $W-47 \mathrm{Ni}$ mezura $R-4^{8}$ Anz satura $C D I K M$, Anz sadura $N S c$, Antz peiura $W$, Ab la dura $R-$ $46-48 N$ ordnet $47,48,46-50$ ri $C D M N R S W$, xi $c$, fehlt $I K-$ 51 Qanz $C D M W$, Anz $1 K$, Tan $C R$, Senz $S c-52$ Sol qe $C D I K M N C W$, sols qe $S$, Qe fos mia $R-53$ Sol un dia $R-54$ fehlt $N R-57$ qatorze] XXIII $C-58 \mathrm{Menz} C$, fehlt $S c-59 \mathrm{E}$ cug sia $M W$, Cre qe sia $C D S c$, uenz cre qe sia $N$, Qe qe sia $R$, Cresia $I K-61-63$ fehlt $S C$ - 69-72 fehlt $S-69$ Lials qe no faill chai $D-71$ On] En $M-$ $73 \mathrm{ab} I K M W$, en $C D$, e $R c$, et $S$; Flor es de verais $N-76$ temenza $I K S c$, tenenza $C D N$, suffrensa $M R W-77$ Qer $N S c$, Qar $M W$, Aus gier $D$, usqier $C R$, Sqer $I K$ 


\section{Conoissensa}

Qui los sieus d'esmai

Trai.

\section{VIII.}

Del maltraire

Mercejaire

85 Sui e fis leals amaire

$E$ no'm vaire

De ben faire.

Qui'm sapcha grazir?

Dir

90 No us aus gaire

Mon vejaire,

Que'l grans beutatz don es maire

M'en fai traire

Mon cor laire.

95 En aquest cossir

Vir.

IX.

Pueys e'm volv e'm vire

En aquest coissire,

Don mais am martire

100

Que d'autra gasainh

Mainh.

X.

Gent gasainha,

Qui que's plainha,

Ma don' Imil' en Romainha

I05 Valor mainha

81 Qi] qeis $N$, qeus $S c$; sieus] sieu $C N-83$ Del] Bel $M$, Selh $C-$ 86 fehlt MW, dafür zwischen 87 und 88: Ses estraire -87 El ben faire $C M W$, Al meu vejaire $R$, De ben faire $D I K N c$, De bon faire $S-$ 88 Cuch $C M W$, Qe $D I K$, Qim $N c$, Cui $S$, Qui $R$; sapcha] sap $R-$ $89-95$ fehlt $S$ - 90 Nous CRSc, non DMNIKW; aus CDIKNSC, lles $M$, laus $W$, say $R-91 \mathrm{Al}$ meu veyaire $R-92$ Qel $C M R S c$, Qilz $W$, La $D$, Las $I K$, El $N$; don es maire] don sui esmaire $N-$ $93 \mathrm{Men} C M S c$, Mi $D I K W, \mathrm{Me} N R$; traire] raire $R-94 \mathrm{Mon}$ ] El meu $R$; cor] cors $W-97 \mathrm{em}$ uolu em uire $I K$, em uolu e uire $C$, uolm em uire $D$, uolu em uire $M$, volg e uire $N$, uolu e uire $R$, uol e uire $S$, uolue em uire $W$, uoil e ueire $c-98$ aquest] tal $R-99 \mathrm{am}] \mathrm{ab} R-$ IO0 dautra $C D I K M N R$, dautre $W c$, daltrel $S-101$ mainh $M W$, fehlt CDIKNRSc - 102 Gent] Ben $M$ - 103 Qi] Cui $c$; qes $C M N S c W$, qen $D I K R$ - $104 \mathrm{Ma}$ donna uelh remagna $C$, Ma doni millon romaingna $D$, Ma dona mil en romaigna $I K$, Ma donna uoluen remainha $M W$, Ma don imil en romagna $N$, Ma donna ab qui remanha $R$, Mi dons na mil en romagna $S c-105$ Ualor] Ualors W 
En que's bainha

Son gai cors plazen

Gen.

XI.

Bella e genta

IIo M'atalenta

Plus qu'al mege fals

Mals.

106 qes $C M N W$, qis $R$, qe $D I K S c$; En] Es $R-107$ Son gacors plazen $C D I K R S c$, Son gai cors plazenz $N$, Sos gais cors cortes $M-$ 108 Gen CDIKRSc, Enz N, Es $M W$ - Zwischen 108 und 109 at $M$ zwei Verse: Plazenz - Genz - 109 Bella e genta CDIKMNRSc, Pzenz genta $W-111$ al] a $S c-112 \mathrm{Mals}] \mathrm{Mal} S c$.

Anmerkung.

Vers 104 geht jedenfalls auf Emilie von Ravenna. Vergl. Einleitig.

\section{Metrisches.}

Das Lied ist ein Descort. Es lälst sich in I I Abschnitte erlegen, die den Namen "Strophen" deshalb verdienen, weil I)der Satz mit dem Abschnitte zu Ende ist, 2) weil alle diese Abschnitte, obgleich nur zum Teil paarweise gleichgebaut, dochalle ein gemeinsames Prinzip im Bau befolgen. Jede Strophe beginnt nämlich mit weiblichem und endet mit männlichem Rene, jede hat überhaupt nur zwei Reime. Die Strophen sind uter sich durch Rims derivatius oder maridatz verknüpft, indemder auslautende Reim jeder Strophe (bis auf einen Fall in Vers or und 102 sogar das ganze Reimwort), der also männlich ist, die folgende Strophe als weiblicher Reim (um I Silbe länger) begint. Das allgemeine Schema ist also $a \cup a \cup \ldots b, b \smile \ldots c, c \cup \ldots d$ (c.

Im Besondern ist das Reimschema folgendes:

I. Str. $4 a \cup 5 a \cup 2 a \cup 5 a \cup 5 b \quad 4 a \cup 5 a \cup 5 a \cup 5 b$

2. Str. $4 b \cup 5 b \cup 2 b \cup 5 b \cup 5 c \quad 4 b \cup 5 b \cup 5 b \cup 5 c$

3. Str. $4 \mathrm{c} \cup 5 \mathrm{~d} 4 \mathrm{c} \cup 5 \mathrm{~d} 4 \mathrm{c} \cup 5 \mathrm{~d} 4 \mathrm{c} \cup 5 \mathrm{~d}$

4. Str. $4 d \cup 5 d \cup 5 d \cup 5 f \quad 4 d \cup 5 d \cup 5 d \cup 5 f$

5. Str. $3 f \cup 3 f \cup 3 f \cup 3 f \cup 3 f \cup 3 f \cup 5 g$ I $g$ $3 f \cup 3 f \cup 3 f \cup 3 f \cup 3 f \cup 3 f \cup 5 g \quad$ Ig

6. Str. $3 g \smile 3 g \cup 3 g \cup 3 g \cup 3 g \cup 3 g \cup 5 h \quad I h$ $3 g \smile 3 g \cup 3 g \smile 3 g \smile 3 g \smile 3 g \cup 5 h \quad I h$

7. Str. $3 h \cup 3 h \cup 5$ i $1 \mathrm{i} 3 \mathrm{~h} \cup 3 \mathrm{~h} \cup 5 \mathrm{i}$ i $\mathrm{i}$ $3 h \smile 3 h \smile 5 \mathrm{i}$ Ii $3 \mathrm{~h} \smile 3 \mathrm{~h} \smile 5 \mathrm{i}$ I i

8. Str. $3 i \smile 3 i \smile 7 i \smile 3 i \smile 3 i \smile 5 k \quad r k$ $3 i \smile 3 i \smile 7 i \smile 3 i \smile 3 i \smile 5 k \quad 1 k$

9. Str. $4 \mathrm{k} \cup 5 \mathrm{k} \cup 5 \mathrm{k} \cup 5 \mathrm{l} \mathrm{Il}$

1o. Str. $3 l \cup 3 l \cup 7 l \cup 3 l \cup 3 l \cup 5 \mathrm{~m}$ I m

II. Str. $3 \mathrm{~m} \cup 3 \mathrm{~m} \cup 5 \mathrm{n}$ I $\mathrm{n}$. 
No. 7.

Ueberlieferung.

Die Canzone Per vós bella dous' amia ist in vier Hss., CDEF, überliefert. CE geben vier Strophen und ein Geleit, DF fünf Strophen und kein Geleit. Schon daraus lälst sich schliefsen, dafs $\mathrm{CE}$ einerseits und $\mathrm{DF}$ andererseits näher zusammengehören. In der That wird uns der Stammbaum:

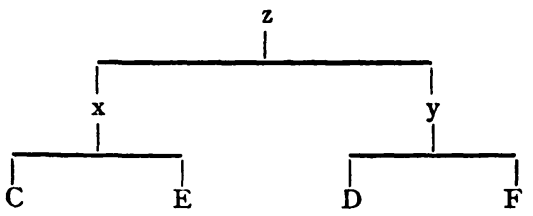

durch folgende gemeinsame Fehler zwischen $\mathrm{C}$ und $\mathrm{E}$ bestätigt:

I 7. In $C$ und $E$ fehlt das non, das man nach dem übergeordneten temenza erwartet und das DF haben. (Merkwürdigerweise druckt es auch Rochegude nicht, obwohl er D oder F noch neben CE hatte, wie das Vorhandensein von Str. 3 bei ihm beweist.)

II 15. In CE fehlt ges; also fehlt auch im Metrum eine Silbe.

$\mathrm{V}$ 52. In CE volria statt volgra; wodurch eine Silbe überzählig wird.

Weder $D$ noch $F$ haben mit einer andern Hs. etwelchen Fehler gemein, aber auch unter sich nicht. Doch können wir aus den vielen Gemeinsamkeiten der Diktion auf näheres Verhältnis schliefsen, besonders da auch beide keine Tornada wiedergeben.

(Orthographie nach $C$, III nach $F$.)

I.

Per vos, bella dous' amia,

Trac nueg e jorn greu martire;

Que d'als no pens ni cossire,

Ans vai doblan tota via

5 L'amors e la bevolensa.

Per qu'ieu ai gran temensa

Que 1 dezirs no m'aucia.

On mais vos bays, doussa res, e vos toc,

Yeu m'en vauc plus prion en aisselh foc.

II.

Io En plus francha senhoria

No pogra mon cor assire;

I Per $D E F$, P.r $C$; amia] . mia $C-2$ nueg e iorn $C D E$, iorn e noich $F$; martire] mar.. re $C-3 \mathrm{Qe}$ dals $C E F$, Ni deus $D$; pens] ...s $C-4$ vai] . ai $C$; via] fehlt $C-5$ Lamors] .....s $C$; beuolensa] bon uolenza $D-6$ Per so ai gran $F$, Pero ai gran $D$, Perquieu ai gran $E$, P.........n $C-7$ no $D F$, fehlt $C E$; dezirs $E F$, dezirier $C$, destriers $D$ - 8 On mais] Qon plus $F$; bays] bai $D-9$ Yeu] Ez eu $F$; vauc] uam $E$; en aisselh $C E$, es $D$, inz el $F-1$ I assire] aissire $D$ 
Qu'ieu non cre qu'el mon se mire

Don'ab tan de cortezia

Ni que de beutat vos vensa.

15

$$
E \text { non ay ges crezensa, }
$$

Per nulha ren que sia

Puesca guerir, s'ieu no complisc lo joc,

$E$ visques tant cum Eli' et Enoc.

\section{III.}

Ai! quantas vez plor lo dia

20 E quantas vez $\mathrm{mi}$ fai rire

L'amors que'm venz e.l desire

E'm destreing lo cor $\mathrm{e} \cdot \mathrm{m}$ lia.

E'l vostr' honrada valenza

$\mathrm{Fez}$ en mon cor semenza

25 Plus que far non solia.

Ara sai eu qu'eu hai begut del broc

Don bec Tristans, qu'anc pois garir no poc.

IV.

Vostr' hom suy ses trichairia

E, si'us platz, podetz m'aucire;

30 Qu'hom non poiria devire

Quill cor del cors no'm trazia.

Que en vos nays e comensa

Beutatz e conoyssensa

Miels qu'hom dir no poiria.

$35 \mathrm{Si} \cdot \mathrm{m}$ destrenhetz mon fin cor en un loc,

Ben a tres ans qu'anc d'un voler no'ys moc.

$\mathrm{V}$.

E ja als jorns de ma via

Non seray d'autra jauzire,

Tant vos suy hom e servire

40 Francx e leyals ses bauzia

Que ses la vostra entendenza

No volgr' aver Proenza

Ab tota Lombardia.

12 se] sen $C-14$ uos] nos $F-15$ E] Pero $D$; ges $F$, falt $C D E$ - 17 complisc] comple $E-18$ E] Sieu $D F$; Eli et $F$, Helos et $C$, elies $D$, Elias et $E-19-27$ fehlt $C E-21$ Lamors $F$, Laras $D-$ 22 Em $F$, En $D-28$ hom $C D E$, oms $F-30$ poiria $C E$, ogra $D$, men pogra $F-31$ cors del cor $D-32$ Quar $C D E F-33$ fehlt $E$; Beltat $D-34$ Miels $C E$, Mais $D F-35$ Qe ten destreig $F$, Jem tenc destreing $D$, Sim destrenhetz $C E$; fin $C E$, ferm $F$, fehlt $D-36$ tres $C E$, cinc $D F$; qanc $C E$, qe $D F$; noys $C$, nos $E F$, nom $D-37$ als iorns $F$, al iorn $C$, a iorn $E$, al iorns $D$; E] Ni $D F-3^{8}$ ion serai dautra $C E$, Dautra non serai $D F-39$ hom $C E F$, oms $D-4$ Qe ses $C E$, Segon $D, E$ ses $F$; entendenza $D E F$, tendenza $C-42$ iolgrauer $D F$, uolriauer $C E$ 
Quan m'auretz dat so don m'aretz dig d'oc,

45 Seray plus rics que'l senher de Marroc.

A l'emperaire agensa

E ten a cortezia,

Quant hom li quer autreyar, e ditz d'oc.

Mas ja als faitz no's pren ren qui no.l loc.

44 mauretz dat $C D F$, mauuesdat $E$; doc $C E$, oc $D F-45$ Serai plus $C E$, Plus serai $D F$; qel $C E F$, del $D-46-49$ fehlt $D F-48$ ditz $C$, $\operatorname{dir} E-49$ pren ren $C$, penrem $E$; nol $C$, no $E-A l s$ Schluss fügt $E$ hinzu: Uol gent guazardonar qan na sazon e loc.

\section{Metrisches.}

Diese einzige erhaltene Canzone Augiers besteht aus fünf Coblas unissonas von je neun und aus einer Tornada von zwei Versen. Jede Cobla zeigt folgendes Reimschema:

$$
7 a \cup 7 b \cup 7 b \cup 7 a \cup 7 c \cup 6 c \smile 6 a \cup 10 d \text { rod. }
$$

Die Strophe ist unteilbar.

Die zehnsilbigen Verse haben alle männliche Cäsur nach der vierten Silbe.

Eine gleichgebaute Strophe findet sich sonst nicht.

No. 8.

Ueberlieferung.

Die Tenzone Guillem, prims iest en trobar a ma guiza ist in den drei Hss. EMR überliefert. Nur $M$ giebt sie ganz, nämlich sechs Strophen und vier Geleite, E hat nur Strophe I, II, V und VI und kein Geleit, R hat sechs Strophen, aber nur zwei Geleite, nämlich III und IV.

$\mathrm{E}$ scheint mit $M$ verwandt, wenigstens deutet dies ein gemeinsamer Fehler an: in $E$ und $M$ fehlt nämlich Vers 3 eine Silbe, welche $\mathrm{R}$ mit sai ausfüllt. Doch hat dieses Verhältnis für die Kritik keine Bedeutung, da gerade die Strophen mit zweifelhaften Stellen (III und IV) von E nicht überliefert werden.

Eine Aenderung gegen alle drei Hss. ist die von Bartsch I, 4 bewirkte Wandelung des volrias in volgras, wodurch der Vers sechssilbig wird, während EMR unregelmälsigerweise sieben Silben zeigen. (Vergl. Bartsch, Chrestom. S. 7 2.)

$$
\text { Joc partit. (EMR). }
$$

(Orthographie nacb $M$.)

I.

Guillem, prims iest en trobar a ma guiza.

Troban vueilh doncs saber

Ta voluntat, pos sai tant l'ajas misa:

I Guillem prims iest $E R$, Guillems prins iestz $M$; guiza $M$, guia $E R$ - uueilh doncs $M$, uolh doncs $R$, uolria $E-3$ sai $R$, fehlt $E M$ 
Qual volgras mais aver:

5 Esser rics de terræ d'aver

Entre'ls plus rics, o la sciënsa priza

$\mathrm{Ab}$ lo saber que las .vii. artz deviça.

II.

Mais volgr' aver la sciënsa conquisa

Que'm degues remaner

Io Que la rictat, que cauza es devisa

Qu'als rics pot pauc valer,

E leu pot hom d'aut bas chazer.

E.l siënça non chai, pos s'es assisa.

Cel qu'all saber es rics en sa camisa.

III.

Ij Cell qu'entre $\cdot 1 s$ rics ha gran ricor pleniera, Que quier d'aqui en sus?

Que .c. savis pot metr'en una tiera

A chascun donan plus.

C'Aristotils sobre'ls prims clus

20 Pres dons de rics e Vergils la ribiera

De Napol jus. Mais am donar que quiera.

IV.

Ges per lenga polida gen parliera,

$\mathrm{N}$ Augier, non lais mon us;

Que sciënsa vai totas ves premiera

25 Tenen los rics enclus.

Car vey que aissi es conclus

Que'l cap del mon fai pujar en cadiera,

E.1 rei Porrus tornet en gran paubrieira.

$\mathrm{V}$.

Guillem, si 1 rics pot perdre manentia

30 Creisser pot atressi,

Per que 1 destrics contra.l creisser pars sia,

E.l segles vai aissi

Que lo rics viu e a la $f i$

Pot dar als sieus tot lo mon, si l'avia,

35 So qu'al savi dieus no volc donar mia.

4 volgras] uolrias $E R$, uolries $M-7$ las vii artz $E M$, la set ars $R-$ Io la rictat $R$, las rictatz $E$, la ricor $M-$ I I Quals rics $M R$, qal ric $E-$ $12 \mathrm{E}$ leu pot hom $E R$, Qar pot hom leu $M$; daut fehlt $E-13 \mathrm{El} M R$, Eill $E$; ses $E M$, es $R-15-27$ fehlt $E-16$ Qe qier daqi en sus $M$, Uol e qier plus en sus $R-18$ Sol ca chascun don plus $R-19$ clus $M$, e lus $R-20$ Uergils $M$, Uirgilis que ditz en $R-21$ De Napol ius $M$, Lay a Napols $R$; mais $M$, may $R-22$ Ges $M$, Guilhem $R-24$ Qe $M$, Qel $R$; totas $M$, tota $R-25$ Tenen $M, \mathrm{E}$ ten $R$; enclus] en clus $M$, conclus $R-26$ Calleissandre venqet porus $R$; es] est $M-27 \mathrm{E}$ sa gran ost el tornet en paubreira $R-28 \mathrm{Ab}$ son saber per qen sec en cadeira $R$ - 29 Guilhem $E R$, Guilhēs $M-31$ Per qel $E R$, E le $M$; pars sia $E$, parsia $M$, pcia $R-32$ vai $E R$, cor $M-33$ Qels rics uiuon tro a la fi $R$; a la fi $M R$, assa fi $E$ 
VI.

L'artz e.l sabers c'Aristotils avia

Viu, si tot el mori,

E porton l'en man prim clerc garentia,

E l'escriptura di

40 C'a Salomo pres enaissi

Qu'el ac lo sen e.l rictat en baillia

Ab lo sen, si fes tot quan far volia.

I.

Aisso que dieus dis ni parti

Non part ieu ges, mas Frans' aver volria

45 E l'emperi mais que saber que sia.

II.

N Augier, no us acordatz à mi

Que paubres es qui mais aver volria.

Sens sobre si non serca manentia.

III.

Sens de naturæ de lati

50 Ha en Romieus, e plai li que rics sia,

E pregem li que d'aquest plag drez dia.

IV.

En Romieus per jujamen di

Que mais val sens que non fai manentia;

Pero à si ditz que l'aver penria.

36 L'artz e'l sabers] Lart el saber $E$, Sens e sabers $M$, Tart el sabers $R$; Aristotils $M R$, Aristotes $E-38$ len man prim clerc] len mains prims clers $E$; len man bon clerc $M$, lin man prim clerc $R-40 Q$ a Salomo $E R$, Qe Salamons $M-41$ Lo sen pueis ac lauer en sa balhia $M-$ $42 \mathrm{Ab}$ lo sen si $E M, \mathrm{Et}$ ab lo sen $R-43-48$ fehlt $R-43-54$ fehlt $E$ - 45 saber] sabers $M-49$ naturae $M$, natura $R-5^{1}$ pregem $R$, pregen $M ;$ drez $M$, uer $R-53 Q$ e be ual mais saber qe manentia $R-$ 54 a si] aissi $M$, assi $R$; ditz qulauer penria $M$, la rictat mais uolria $R$.

\section{Anmerkungen.}

I 4. Bartsch bessert hier volrias zu volgras.

III 16. Soweit wie möglich folge ich $M$, weil dies in unsicheren Fällen das Richtigere hat.

III 2O-2I. Bartsch murs $R$ verändern und auf den Binnenreim, den er vielleicht übersehen hat, mit $R$ verzichten. Das Natürlichere und $R$ ich. tigere (mit Binnenreim) gielt hitr M. S. Metrisches. E Vergils la ribeira de Napol jus bezieht sich jedenfalls auf das Landgut bei Neapel, das Augustus dem Vergil schenkte.

IV 26-28. Auch hier verfehlt Bartsch mit $R$ den Binnenreim in Vers 28. Darum ist auch hier $\mathbf{M}$ vorzuziehen. Doch muls in Vers 26 est (M) in es gewandelt werden.

Tornada IV 54. Bartsch ändert das aissi von $\mathrm{M}$ und assi von $\mathrm{R}$ in a si. (Bartsch, Chrestom. S. 72.) 


\section{Metrisches.}

Dies Gedicht ist eine Tenzone, näher ein Joc partit. Es besteht aus Coblas doblas, wie das ja für Tenzonen sehr natürlicth ist. Im ganzen sind es 54 Verse, nämlich sechs Coblas von jie sieben Versen und vier Tornadas von je drei Versen.

Strophenschema:

$$
10 a \cup 6 b \text { 10a } \cup 6 b 8 b \text { roa } \cup 10 a \cup
$$

oder genauer:

$$
10 a \smile 6 b \text { roa } \smile 6 b \quad 8 b \quad \text { roa } \underbrace{4 b+6 a}
$$

Der letzte Zehnsilbner hat Binnenreim vor der Cäsur, dieser Binnentreim ist stets männlich (b). In den übrigen Zehnsilbnern findett sich teils männliche teils lyrische Cäsur.

Bartsch hat in seiner Ausgabe dieses Gedichtes den Binnenreim nicht beachtet, wo Hs. $\mathrm{R}$ ihn nicht giebt, also in Strophe IIII und IV. Ich gebe diese Strophen nach M, welches den Binnenreim in allen Strophen durchführt.

Die Strophe ist nicht in pedes und versus teilbar.

No. 9 .

Tenzone. $(E$.)

I.

Bertran, vos c'anar soliatz ab lairos

Panan bueus e box e cabras e moutos,

Porcx e galinas et aucas e capos,

Eras, glotz e raubaire,

Diguatz vostre vejaire:

Qual mestiers es plus aontos:

D'esser jotglar ho laire?

IIn.

N Augier, cascus mi par crois et enujos,

E trac ne vos a guiren, que d'ambedos

Io Es sages, e retengues que's tanh a vos;

Que, per esser chantaire,

Laisses lansar e traire;

$E$ jotglars pren aunitz dos,

Es sirvens de donaire.

III.

I5 Bertran, mestier no m'azauta de sirven;

C'om l'espesa e l'eisorba e l'art ell pen,

E jotglar sercan baros e gaia gen

E vivon az onranza.

Bar, s'aisso par mermansa,

Tu t'en torna, si t'es gen,

A la lor benanansa. 
IV.

N Augier, trop etz envejos d'anar quiren, Quar de vos home aun fag veill vestimen,

Et ieu vueill tolre e raubar, que m'er plus gen,

$E$ portar dart e lansa;

Que aisi viu hom en Fransa.

C'a vos pot cel dar colps sen

Qui be'us umplis la pansa.

V.

Bertran, can ieu vauc sercan gaug e solatz,

30 E vos toletz e raubatz tan tro.l pecatz

Vos meta en mas de vilas ab poinhs liatz

En mercatz ho en feira,

On hom vos frust e'us feira;

Pueis quan seretz ben frustatz,

35

Traira.us hom la lumneira.

VI.

N Augier, tost auretz trobat so que sercatz,

Qu'ab cascun intras manjar descovidatz.

Calacom portier vos er mal ensenhatz,

Que'us fara com Calveira,

Jotglar, boca parleira,

C'a vos fe, so que m'oratz,

La jos en la carreira.

Anmerkungen.

Diese Tenzone ist nur in Hs. $\mathrm{E}$ überliefert und zwar mangelhaft. Folgende Aenderungen gegen $\mathrm{E}$ waren notwendig:

14 sel ques tanh de uos (E) zu: que's tanh a vos - $14 E$ siruens es de donaire (E) $\mathrm{zu}$ : Es sirvens de donaire - 19 si aisso (E) zu: s'aisso 22 trop enueios (E) zu: trop ets envejos - 23 Quar de uos aun fag weill uestimen (E) vielleicht zu: Quar de vos home aun fag veill vestimen 27 pot dar (E) zu: pot cel dar - 29 ieu uauc (E) zu: can ieu vauc 35 sa lumenaira (E) zu: la lumneira; traire la l. bedeutet "blenden“. 39 Qeus fara calueira (E) wurde geändert zu: Que'us fara com Calveira - und zwar auf den Vorschlag von Herrn Professor Suchier, welcher in dem sonst nicht belegbaren calveira einen Spottnamen auf Guillem Figueira (vergl. Einleitung) sieht, da die gleiche Silbcnzahl und Endung der beiden Worte einen solchen Spottnamen nahe legen. Anzunehmen wäre dann, dafs Figueira kahlköpfig gewesen sei; denn calveira mufs doch wohl mit calvus „kahl“ zusammenhängen. - 4I $A$ vos (E) ist hier geändert zu: $C^{\prime} a$ vos auf Vorschlag von derselben Seite. Die Stelle spielt danach auf Prügel an, die Augier von Figueira vielleicht als Strafe für den Schwertstreich (vergl. Einleitung) erhalten hat.

\section{Metrisches.}

Das Gedicht ist eine Tenzone, genauer ein Joc partit. In allen Strophen mufste der überlieferte Text des Versmaises wegen Aenderungen erfahren. Wie die Mehrzahl der Tenzonen besteht auch diese aus Coblas doblas; im ganzen sind es 42 Verse, nämlich sechs Coblas zu je sieben Versen. 
Schema: II a II a I a $6 \mathrm{~b} \cup 6 \mathrm{~b} \cup 7 \mathrm{a} 6 \mathrm{~b} \cup$.

Ueber diese Strophenform handelt Bartsch (Ztschr. f. rom. Phil. II, 197) und stellt über den Elfsilbler in dieser Strophe folgende Regeln auf: „Die Cäsur kann männlich und weiblich sein. Elision über Cäsur ist erlaubt; bei männlicher Cäsur muls die zweite Vershälfte immer vier Silben haben."

In der etwas veränderten Gestalt

$$
\text { I I I I a I I a } 6 \mathrm{a} \smile 6 \mathrm{~b} \smile 7 \mathrm{x} 6 \mathrm{~b} \smile
$$

tritt die obige Strophe in einer Tenzone zwischen Guillem Figueira und Aimeric de Peguilhan auf, und in allen drei Tenzonen sind die letzten Reime der Strophen dieselben.

Weil diese seltene Strophenform bei diesen beiden Joglars vorkommt, dürfen wir wohl eine nähere Beziehung Augiers zu ihnen annehmen und unsern Joglar mit Paul Meyer (Rom. X, 252) als identisch mit jenem Auzer annehmen, der dem Figueira den Schwertstreich versetzte (vergl. Einleitung). Schon Suchier (Jahrb. 14, 292) hatte vor Meyer die enge Beziehung der drei Tenzonen an dem so ähnlichen Bau erkannt und auch den Bertran der Tenzone mit dem Bertran d'Aurel identifiziert, der in dem Kreise Guillem Figueiras und Augiers verkehrte.

Johannes Müller. 